\section{俩 Heighten Science \\ P U B L I C I T I O N S Corporation ISSN 2639-6653}

\title{
Ontario wait time strategy to solve long waiting problem
}

\author{
Satyajit Patra* \\ Associate Professor, American International Medical University, St. Lucia
}

*Address for Correspondence: Satyajit Patra, $\mathrm{PhD}$, Associate Professor, American International Medical University, St. Lucia, Email:

dr.patra@aimu-edu.us

Submitted: 12 October 2018

Approved: 26 October 2018

Published: 27 October 2018

Copyright: ๑ 2018 Patra S. This is an open access article distributed under the Creative Commons Attribution License, which permits unrestricted use, distribution, and reproduction in any medium, provided the original work is properly cited

Check for updates
The Ontario government has been battling with the issue of extensive wait times in hospitals for several years. Although there are many complex reasons that stem off of the issues of wait times, such as available in patient beds and bed blockers (patients who stay in the hospitals for long periods of time after sustaining a massive injury), the primary cause is concentrated as a system wide problem in access to care [1]. Through numerous reforming and restructuring plans, the Ontario government devised a Wait Time Strategy plan to monitor, manage and improve access to health care services including surgeries and time spent in the ER. The strategy was also devised to enhance the efficiency and effectiveness of healthcare provision. The information derived from the results of the Ontario Wait Time Strategy (OWTS) was to be made public to citizens and providers to ensure that everyone is well aware of the results. Yet, it is quite difficult to implement such a strategy if the leadership challenges within the hospital are not addressed [2].

Accountability is seen as a key driver to success in healthcare in order to initiate and implement change. There is a need to shift from a few individuals responsible, to an environment where multiple levels and types of individuals are accountable for accomplishing a wide range of results. Canadians have stressed the need for greater accountability in the healthcare system, yet achieving accountability and assigning who is responsible when things go wrong is a lurking issue in healthcare. Hospital leaders need to adapt an infusion management strategy, where everyone is responsible for change and its adverse events [3].

Furthermore, there are various work roles that are involved in a single hospital that all need to be aligned in order to effectively provide quality care. Hospitals and physicians operate as distinct units; for instance, hospital decisions are conducted through committees, authorities and boards whereas physician practices are led through chief physicians and other clinical leaders. Physicians do not work in teams and due to this division of specialization and standardization of knowledge and skills, all appointments and procedures leading up to surgery are managed individually, leading to delays and inefficiencies at every step. This segregation between services is seen between hospitals and community settings that are enlightened through the Ontario wait time strategy.

Hospital leaders are under tremendous scrutiny to conform to the needs of major stakeholders, tackle the various changes imposed by the Ministry of Health, where the endless list of duties puts severe strains on the effectiveness of efficient healthcare leadership. Although there is no specific indicator to measure the success and competence of leadership, measures such as wait times can be used to assess health system performance, in correlation with operative healthcare leadership. With close monitoring and incremental improvements in leadership and integration, through time, there will be a decrease in wait time and an overall improvement in Canadian health care which will transcend generations. 
The government took strategic action by increasing the funding initiatives for procedures accounting about 240,000 additional procedures to improve health care, with emphasis on surgical procedure and record increase in the number of procedures in decades. However the increase in number is not the simple answer, the effective solution is to do in a smarter way. To make more accessible of the information to the public, a web site was lunched by the government. This has detailed information of periodically updated data on wait times with information from specific hospitals across Ontario.

It is obvious that the changes made by the government will ensure treatment much sooner, not only it will allow the resident of Ontario to keep updated increased demand of population ages, also in longer term will help in shortening the wait time. Public with unceasing longer waiting time and increased frustration are now seeing relief and coming forward with strong support of the new modifications in the waiting time in the clinics, at the same time the ministry is also committed to enhancing the transformation of heath care system to next level.

\section{References}

1. Gaughan J, Gravelle H, Santos R, Siciliani L. Long-term care provision, hospital bed blocking, and discharge destination for hip fracture and stroke patients. Int J Health Econ Manag. 2017. Ref.: https://goo.gl/Qfs1aE

2. Bruni RA, Laupacis A, Levinson W, Martin DK. Public views on a wait time management initiative: a matter of communication. BMC health services research. 2010; 10: 228. Ref.: https://goo.gl/7R6ZK9

3. Duckett S, Kempton A. Canadians' Views about Health System Performance. Healthcare policy = Politiques de sante. 2012; 7: 85-101. Ref.: https://goo.gl/nQuYY3 